\title{
APLICAÇÃO DAS TÉCNICAS DE IDENTIFICAÇÃO DE RISCO EM EMPREENDIMENTOS DE E \& P
}

\author{
Cássia Andréa Ruotolo Morano ${ }^{1}$ \\ Claudia Garrido Martins ${ }^{2}$ \\ Miguel Luiz Ribeiro Ferreira ${ }^{3}$
}

\begin{abstract}
Resumo: O gerenciamento de risco apresenta uma importância cada vez maior no cenário mundial, e vem sendo aplicado na última década de forma crescente por grandes empresas brasileiras. De uma forma geral o modelo adotado segue o preconizado pelo "PMI - Project Management Institute”. Entretanto, em relação à indústria da construção, este assunto está em desenvolvimento. Deste modo, verifica-se que tanto a realização do gerenciamento de risco em projetos, quanto os resultados por ele gerado ainda são pouco conhecidos. Assim sendo, o objetivo deste trabalho é descrever a etapa de identificação de risco, que se constitui em um dos processos do ciclo do gerenciamento de risco, mostrando quais as técnicas mais utilizadas e como a técnica "Brainstorming" foi aplicada em três empreendimentos na área de exploração e produção de petróleo (E \& P), comparando os resultados obtidos entre os projetos e a literatura.
\end{abstract}

Palavras-chave: Gerenciamento de risco, identificação de risco, Brainstorming.

Abstract: Nowadays, the risk management shows a greater importance in the worldwide scenery, and big Brazilian companies have applied it in the last decade in a growth manner. In general, the model adopted follows the PMI - Project Management Institute processes description. Meanwhile, related to construction industry, this is a development subject. In this manner, the purpose of this work is to describe the risk identification phase, as one of the risk management processes, describing what the more used risk identification techniques are and how the Brainstorming technique was applied in three enterprises in the exploration and production petroleum area ( $\mathrm{E} \& \mathrm{P}$ ), projects, comparing the attained results between those projects and the literature.

Keywords: Risk Management, Risk Identification, Brainstorming.

\section{INTRODUÇÃO}

Verifica-se no Brasil que existe um grande aumento do interesse sobre o gerenciamento de risco em projetos, principalmente entre as grandes construtoras, a indústria naval e offshore. Isto ocorreu principalmente devido à difusão das melhores práticas para gerenciamento de projetos proposta pelo "PMI - Project Management Institute", que estabelece como uma de suas áreas de conhecimento o gerenciamento de risco. (PMBOK-PMI, 2004). Entretanto, de acordo com o trabalho realizado por Morano (2003) o gerenciamento de risco, no que diz respeito à implantação de projetos de construção, vem sendo aplicado de forma incipiente na indústria da construção brasileira, nos seus mais variados ramos. Por outro lado, segundo

\footnotetext{
${ }^{1}$ Programa de Pós-Graduação em Engenharia Civil/UFF - crmorano@poscivil.uff.br

${ }^{2}$ MBA em Engenharia Econômica e Financeira/UFF - LATEC - cgarrido@pobox.com

${ }^{3}$ Programa de Pós-Graduação em Engenharia Civil/UFF - temmlrf@vm.uff.br
} 
BACCARINI (2001), tem havido um crescimento constante da prática deste gerenciamento que passou a ser um elemento importante no processo de tomada de decisão dentro do gerenciamento de projeto. Todavia, observa-se que existem inúmeras técnicas de identificação, avaliação e análise de risco na literatura que auxiliam e fazem parte de todo este processo e que não são aplicados como o estabelecido ou propostos pelos autores. Da mesma forma, Akintoye \& Macleod (1997) apud. (MARTINS, 2006, p. 31) menciona que o gerenciamento de risco tem sido aplicado recentemente pelas indústrias da construção, de defesa e petróleo. Entretanto, os resultados obtidos e as técnicas empregadas neste gerenciamento e na análise de risco ainda são poucos conhecidos.

No presente caso, o objetivo deste trabalho é o de apresentar o processo de identificação de risco e as técnicas mais utilizadas em projetos. Da mesma forma, mostrar como foi aplicada a técnica de identificação de risco "Brainstorming" em três empreendimentos na área de exploração e produção de petróleo (E \& P) e promover a comparação desta aplicação com a literatura a fim de se verificar as convergências e divergências existentes.

Para o desenvolvimento desta pesquisa realizaremos a análise documental de três projetos de engenharia, construção e montagem de plataformas.

\section{GERENCIAMENTO DE RISCO}

O modelo de gerenciamento de risco que vem sendo adotado pelas grandes empresas construtoras das mais diversas áreas é o do "PMI", cujo propósito é a aplicação de conhecimento, habilidades, ferramentas e técnicas às atividades do projeto a fim de que sejam atendidos os objetivos estabelecidos. Deste modo, o gerenciamento de risco consiste no planejamento, identificação, análise, respostas, monitoramento e controle dos ENGEVISTA, v. 8, n. 2, p. 120-133, dezembro 2006 riscos em um projeto, sendo que o seu principal objetivo é o de aumentar a probabilidade e o impacto dos eventos positivos e minimizar a probabilidade e 0 impacto dos eventos adversos ao projeto. (PMBOK-PMI, 2004).

No trabalho realizado por Morano (2003) verificou-se que de uma forma geral, as metodologias de gerenciamento de risco seguem a seguinte divisão: identificação, análise ou avaliação, ações de prevenção e controle ou monitoramento.

\section{IDENTIFICAÇÃO DE RISCO}

De uma forma geral, observa-se na literatura que os autores consideram a fase de identificação de risco como uma das mais importantes em todo processo do gerenciamento de risco, pois apresenta um impacto maior na acuracidade das avaliações de risco, já que a forma como os riscos são identificados e coletados constituem-se na questão central para a efetividade de todo este processo. (MARTINS, 2006). Da mesma forma, (KERZNER, 1998) menciona que o primeiro passo para identificar os riscos é detectar as áreas potenciais de risco, sendo que através da eficácia desta identificação resultará a eficiência do gerenciamento de risco.

Segundo o PMBOK (PMI, 2004), a fase de identificação de risco compreende a determinação de quais riscos podem afetar o projeto e em documentar as suas características.

De maneira semelhante, Baccarini (2001) define a identificação de risco como "o processo de determinar o que pode acontecer, porque e como".

De acordo com Chapman, R. J. (1998), a identificação dos riscos pode ser dividida em três categorias:

- A identificação de risco, conduzido somente pelo analista de riscos baseando-se apenas em sua experiência, conhecimento e habilidade, sendo que este especialista levará em conta a 
revisão do ciclo de vida do projeto e os dados históricos da organização;

- A identificação de risco, conduzida através da entrevista do analista de riscos com um ou mais membros da equipe de projeto, analisando também os dados históricos e o ciclo de vida do projeto, e baseando-se no conhecimento e experiência dos profissionais que forem entrevistados;

- A identificação de risco, onde o analista de riscos lidera um ou mais grupos de trabalho, utilizando as técnicas de identificação de risco.

Por outro lado, devido à fase de identificação de risco corresponder a um dos processos do ciclo de gerenciamento de risco, pressupõe que existam entradas e que sejam produzidas saídas. Deste modo, o diagrama da Figura 1 exemplifica os itens que compõem o processo de identificação de risco.

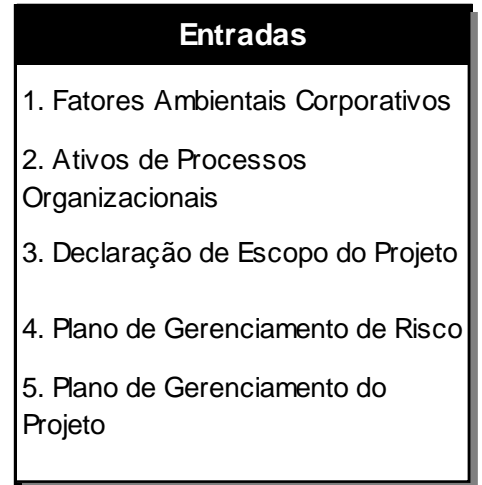

\section{Ferramentas e Técnicas}

1. Revisões da Documentação

2. Técnicas de Captura de

Informação

3. Análise de Checklists

4. Análise de Premissas

5. Técnicas de Diagramação

\section{Saídas}

1. Registro do Risco

Figura 1 - Identificação de Risco: Entradas, Ferramentas e Técnicas, e Saídas. Fonte: (PMBOK, 2004, p. 246)

De acordo com PMBOK - PMI (2004) verifica-se que as entradas do processo de identificação de risco englobam os seguintes pontos:

1. Fatores Ambientais Corporativos - São fatores da empresa que circundam e influenciam o sucesso do projeto, e que desta forma devem ser considerados. Tais fatores incluem, mas não se limitam a: estrutura e cultura organizacional, tolerância dos envolvidos no projeto ao risco, padrões da indústria, condições de mercado, sistemas interno de trabalho.

2. Ativos de Processos Organizacionais São políticas, padrões, processos, modelos, guias ou requisitos que fazem parte do ativo do conhecimento e aprendizado da organização.

3. Declaração de Escopo do Projeto - é utilizada basicamente neste processo para que as premissas encontradas neste documento sejam avaliadas quanto ao seu grau de incerteza e como potenciais causas de risco.

4. Plano de Gerenciamento de Risco - é usado basicamente para a consulta das definições de atribuições e responsabilidades, e das categorias de risco.

5. Plano de Gerenciamento do Projeto Através deste plano revisam-se as saídas das demais áreas do gerenciamento do projeto para identificação de possíveis riscos, além do entendimento dos planos de gerenciamento de áreas críticas tais como prazo, custo e qualidade.

Da mesma forma, as Ferramentas e Técnicas abordam os seguintes pontos:

1. Revisões da Documentação - Procedese a uma revisão estruturada da documentação gerada no projeto 
(planos, premissas, documentos e informações arquivadas) em busca de indicadores de risco.

2. Técnicas de Captura de Informação São técnicas estruturadas que auxiliam na captura individual ou em grupo de informações.

3. Análise de "Checklists" - "Checklists" de identificação de risco geradas a partir de informações históricas e conhecimentos acumulados de projetos anteriores similares.

4. Análise de Premissas - é uma ferramenta que explora a validade das premissas aplicadas ao projeto, baseada na falta de acuracidade, inconsistência e falta de complementação destas.

5. Técnicas de Diagramação - São técnicas gráficas que auxiliam no levantamento e compreensão de possíveis riscos.
Em relação às Saídas teremos como resultado o registro do risco que irá conter as características principais de cada um dos riscos identificados, incluindo sua descrição, categoria, e causas. Este registro é atualizado nas demais fases do gerenciamento de risco.

\section{TÉCNICAS DE IDENTIFICAÇÃO DE RISCO}

Observa-se através do levantamento bibliográfico realizado a existência de várias técnicas propostas para a identificação de riscos em projetos.

Segundo a literatura pesquisada o Quadro 01 a seguir especificado apresenta de forma resumida as técnicas de identificação de risco mais usuais em projetos.

\begin{tabular}{|c|c|c|}
\hline $\mathrm{N}^{0}$ & Técnica & Descrição \\
\hline 1 & "Brainstorming" & $\begin{array}{l}\text { Técnica de geração de idéias em grupo dividida em duas fases: (1) fase criativa, } \\
\text { onde os participantes apresentam o maior número possível de idéias (2) fase crítica, } \\
\text { onde cada participante defende sua idéia com o objetivo de convencer os demais } \\
\text { membros do grupo. Na segunda fase são filtradas as melhores idéias, permanecendo } \\
\text { somente aquelas aprovadas pelo grupo. A técnica é composta de quatro regras } \\
\text { básicas: (1) As críticas devem ser banidas - a avaliação das idéias deve ser } \\
\text { guardada para momentos posteriores; (2) A geração livre de idéias deve ser } \\
\text { encorajada; (3) Foco na quantidade - quanto maior o número de idéias, maiores as } \\
\text { chances de se ter idéias válidas; (4) Combinação e aperfeiçoamento de idéias } \\
\text { geradas pelo grupo. (BACCARINI, 2001); (CHAPMAN, 1997); (CHAPMAN, } \\
\text { 1998); (CHAPMAN, 2001); (DEY \& OGUNLANA, 2004); (KERZNER, 2001); } \\
\text { (MORANO, 2003); (PMBOK - PMI, 2004); (RAMP, 2006); (TURNER apud } \\
\text { DEY, 2001); (UHER \& TOAKLEY, 1999) (NÓBREGA et al, 1997) (OSBORN } \\
\text { apud CHAPMAN, 1998) }\end{array}$ \\
\hline 2 & $\begin{array}{l}\text { 'Brainstorming' } \\
\text { Eletrônico }\end{array}$ & $\begin{array}{l}\text { O 'Brainstorming' eletrônico tem por objetivo gerar idéias via web, ou seja, através } \\
\text { de computadores ligados em rede, onde os participantes terão acesso mais rápido } \\
\text { das idéias geradas e podendo desenvolver novas idéias. (Aiken et al., 1994). Esta } \\
\text { técnica corresponde uma abordagem aprimorada do 'Brainstorming' tradicional, } \\
\text { garantindo o anonimato entre os participantes e uma similaridade à equipe de } \\
\text { trabalho, uma vez que não haverá influência ou monopólio de um participante em } \\
\text { relação ao grupo, contribuindo para superação dos problemas gerados devido às } \\
\text { diferenças de hierarquia, experiência e conhecimento de alguns em relação aos } \\
\text { outros membros da equipe; Possibilita a comunicação paralela, permitindo aos } \\
\text { participantes entrar com os comentários simultaneamente e contribuir com novas idéias, } \\
\text { sendo que devido ao grande número de informações geradas, o grupo participante poderá ser } \\
\text { maior; Automatização dos registros, permitindo que todos os comentários e idéias gerados } \\
\text { pela equipe participante sejam armazenados. (MORANO, 2003). }\end{array}$ \\
\hline 3 & Técnica Delphi & $\begin{array}{l}\text { “... 'Delphi' é uma técnica para a busca de um consenso de opinião de um grupo de } \\
\text { especialistas a respeito de eventos futuros. (...) baseia-se no uso estruturado do } \\
\text { conhecimento, da experiência e da criatividade de um painel de especialistas, pressupondo-se } \\
\text { que o julgamento coletivo, quando organizado adequadamente, é melhor que a opinião de } \\
\text { um só indivíduo”. (Wright \& Giovinazzo, 2000, p.54) } \\
\text { Esta técnica de criação de consenso utiliza respostas escritas ao invés de reunir } \\
\text { pessoalmente os membros do grupo, ou ainda método para a sistemática coleta e }\end{array}$ \\
\hline
\end{tabular}




\begin{tabular}{|c|c|c|}
\hline & & $\begin{array}{l}\text { comparação crítica de julgamentos, de participantes anonimamente isolados, sobre } \\
\text { um tópico, através de um conjunto de questionários cuidadosamente desenvolvidos, } \\
\text { intercalados com informações sumarizadas e “feedback” das opiniões, derivadas } \\
\text { das respostas anteriores. (CHAPMAN, 1998); (CHAPMAN, 2001); (DEY \& } \\
\text { OGUNLANA, 2004); (DEY apud DEY, 2001); (KERZNER, 2001); (MORANO, } \\
\text { 2003); (PMBOK - PMI, 2004) (DELBECQ et al. Apud BERUVIDES, 1995) } \\
\text { (CHAPMAN, 1998) }\end{array}$ \\
\hline 4 & $\begin{array}{l}\text { Entrevista/ } \\
\text { Julgamento de } \\
\text { Especialistas }\end{array}$ & $\begin{array}{l}\text { Entrevistas livres, semi-estruturadas ou estruturadas conduzidas individualmente ou } \\
\text { em grupo com membros experientes do projeto, envolvidos ou especialistas. } \\
\text { (BACCARINI, 2001); (CHAPMAN, 1997); (CHAPMAN, 2001); (KERZNER, } \\
\text { 2001); (PMBOK - PMI, 2004); (RAMP, 2006); (TURNER apud DEY, 2001) }\end{array}$ \\
\hline 5 & $\begin{array}{l}\text { Identificação de } \\
\text { Causa }\end{array}$ & $\begin{array}{l}\text { Processo desenhado usado na investigação e categorização das causas essenciais de } \\
\text { um risco, sendo dividida em quatro etapas, a saber: coleta de dados; diagramação } \\
\text { do fator de causa; identificação da causa raiz e geração da recomendação e } \\
\text { implementação. (PMBOK - PMI, 2004) (ROONEY \& HEUVEL, 2004) }\end{array}$ \\
\hline 6 & $\begin{array}{l}\text { Análise SWOT } \\
\text { (“strengths”, } \\
\text { "weaknesses”, } \\
\text { “opportunities", } \\
\text { "threats”) }\end{array}$ & $\begin{array}{l}\text { Acrônimo para "Strengths, Weakness, Opportunities and Threats", que em } \\
\text { português podemos traduzir como Forças, Fraquezas, Oportunidades e Ameaças. É } \\
\text { uma ferramenta de planejamento estratégico, utilizada para análise de projetos e/ou } \\
\text { negócios, ou em qualquer outra situação que envolva uma decisão. A aplicação da } \\
\text { técnica consiste na avaliação do projeto sob cada uma das quatro perspectivas: } \\
\text { forças, fraquezas, oportunidades e ameaças, geralmente apresentadas em forma de } \\
\text { quadrantes. (MARTINS, 2006) (WIKIPEDIA, 2006) (PMBOK - PMI, 2004) }\end{array}$ \\
\hline 7 & “Checklist” & $\begin{array}{l}\text { Consiste em uma lista de itens, que vão sendo marcados como sim ou não, podendo } \\
\text { ser utilizada por um membro da equipe, em grupo ou em uma entrevista. } \\
\text { (AKINTOYE \& MACLEOD, 1997); (BACCARINI, 2001); (CHAPMAN, 1997); } \\
\text { (PERRY \& HAYES apud DEY, 2001); (PMBOK - PMI, 2004); (RAZ \& } \\
\text { MICHAEL, 2001); (UHER \& TOAKLEY, 1999) }\end{array}$ \\
\hline 8 & $\begin{array}{l}\text { Diagrama de } \\
\text { Causa e Efeito }\end{array}$ & $\begin{array}{l}\text { O Diagrama de Causa e Efeito é também conhecido como Diagrama de Ishikawa } \\
\text { ou Espinha-de-peixe, e é útil para a identificação da causa dos riscos. O diagrama é } \\
\text { montado organizando o efeito à direita e as causas à esquerda. Para cada efeito } \\
\text { existem categorias de causas. As causas principais podem ser agrupadas por estas } \\
\text { categorias. (PMBOK - PMI, 2004) (MARTINS, 2006) (UHER \& TOAKLEY, } \\
\text { 1999) }\end{array}$ \\
\hline 9 & Fluxograma & $\begin{array}{l}\text { Representação gráfica que apresenta os passos de um processo. Assim, esta técnica } \\
\text { é aplicada para compreender como os riscos, ou os elementos de um sistema se } \\
\text { interrelacionam. (CHAPMAN, 2001) (PMBOK - PMI, 2004) }\end{array}$ \\
\hline 10 & $\begin{array}{l}\text { Diagrama de } \\
\text { Influência }\end{array}$ & $\begin{array}{l}\text { Representação gráfica contendo nós que representam as variáveis da decisão de um } \\
\text { problema. Um diagrama de influência tradicional é composto por três tipos de nós: } \\
\text { decisão, incerteza, resultado; e por dois tipos de relação entre os nós: relação causal } \\
\text { e relação de precedência. A relação causal ocorre entre os elementos de resultado e } \\
\text { de incerteza e indica uma dependência probabilística. A relação de precedência } \\
\text { ocorre entre elementos de decisão e representam precedência de tempo. } \\
\text { (CHAPMAN, 2001); (DEY \& OGUNLANA, 2004); (KERZNER, 2001); (PMBOK } \\
\text { - PMI, 2004); (RAZ \& MICHAEL, 2001); (UHER \& TOAKLEY, 1999); } \\
\text { (WILLIAMS, 1995) (CROWLEY, 2004) (JOHNSON et al., 2006) }\end{array}$ \\
\hline 11 & $\begin{array}{l}\text { Técnica de Grupo } \\
\text { Nominal }\end{array}$ & $\begin{array}{l}\text { A técnica o grupo nominal foi elaborada para ser utilizada na área de planejamento, } \\
\text { com o objetivo de ampliar a produção criativa do grupo, facilitar as decisões em } \\
\text { equipe, estimular a geração de idéias críticas e servir como instrumento de } \\
\text { agrupamento de idéias. Assim sendo, esta técnica corresponde na geração } \\
\text { silenciosa de idéias escritas; Exposição das idéias geradas ao grupo na forma de } \\
\text { frases simples em cartões ou tiras de papel; Discussão de cada idéia registrada para } \\
\text { esclarecimento e avaliação; Votação individual das idéias em ordem de prioridade, } \\
\text { com a decisão do grupo sendo trabalhada matematicamente através da classificação } \\
\text { por quantidade de votos obtidos ou ordenação por ordem de prioridade. } \\
\text { (CASSIANI \& RODRIGUES,1996) (DELBECQ apud CHAPMAN, 2001) } \\
\text { (CHAPMAN, 1998); (KERZNER, 2001); (MORANO, 2003) }\end{array}$ \\
\hline 12 & "Pondering" & $\begin{array}{l}\text { Abordagem simples e muito básica, que envolve uma só pessoa para identificar os } \\
\text { riscos, e pode servir como uma opção padrão quando nenhuma outra abordagem é } \\
\text { possível. Entretanto, faz-se necessário que a pessoa tenha vivência e experiência na } \\
\text { área onde estão sendo identificados os riscos. Na aplicação desta técnica a pessoa }\end{array}$ \\
\hline
\end{tabular}




\begin{tabular}{|c|c|c|}
\hline & & $\begin{array}{l}\text { sozinha reflete, pondera ou considera o problema, gerando a lista de opções. } \\
\text { (CHAPMAN \& WARD apud BACCARINI, 2001) }\end{array}$ \\
\hline 13 & Sinética & $\begin{array}{l}\text { O objetivo desta técnica é resolver problemas de forma criativa. Deste modo seu } \\
\text { foco consiste em unir objetos e idéias aparentemente diferentes e irrelevantes. } \\
\text { Assim sendo, é proposto o uso de elementos sem conexão em suas analogias ou o } \\
\text { uso de metáforas para gerar a compreensão do problema. } \\
\text { Os participantes devem ter como atribuições essenciais na aplicação desta técnica } \\
\text { uma grande imaginação e conviç̧ão em seus pontos de vista, uma vez que terão } \\
\text { que fazer a conexão do problema com a metáfora. Por outro lado, o uso de metáfora } \\
\text { quebra a opinião do grupo, uma vez que haverá divergência na sua associação com } \\
\text { o problema em questão. Entretanto, o desafio do grupo está em identificar somente } \\
\text { os aspectos positivos em que a metáfora poderá ser aplicada e desenvolver a } \\
\text { construção de alternativas para resolução do problema ou questão. De um modo } \\
\text { geral, as regras de uma sessão de Sinética são similares as de uma sessão de } \\
\text { "Brainstorming”, inclusive com a presença de um facilitador para conduzir a } \\
\text { sessão. (HALL, 1996); (MORANO, 2003); (RAMOS, 2006); (SABA, 2006) }\end{array}$ \\
\hline 14 & $\begin{array}{l}\text { Criação de } \\
\text { Cenários }\end{array}$ & $\begin{array}{l}\text { Caracteriza-se pelo desenvolvimento de cenários hipotéticos, que representam os } \\
\text { processos a serem desenvolvidos, através da construção lógica de cada um dos } \\
\text { eventos e suas interações, e seus resultados gerados. } \\
\text { Ao analisar o cenário do projeto, verificar se existe elementos de risco que possam } \\
\text { ocorrer simultaneamente e se as variações entre eles são altas ou baixas; } \\
\text { Identificar o condutor do risco, ou seja; a causa de um grupo de variáveis do } \\
\text { conjunto, gerar altos ou baixos riscos; Planejar um cenário com variáveis de } \\
\text { incerteza e correlacioná-las, computando os seus impactos no empreendimento; } \\
\text { Identificar os fatores de risco, como por exemplo, uma dificuldade tecnológica, } \\
\text { uma estimativa otimista demais ou uma possível tensão do mercado de trabalho, } \\
\text { entre outros; Computar os impactos causados pelos condutores de risco em relação } \\
\text { ao objetivo do projeto; Combinar a ocorrência de possíveis eventos e correlacioná- } \\
\text { los entre si, através das técnicas de simulação. (MORANO, 2003); (UHER \& } \\
\text { TOAKLEY, 1999); (MARTINS, 2006) }\end{array}$ \\
\hline 15 & Questionário & $\begin{array}{l}\text { Perguntas ao nível do atributo/característica com dicas/exemplos específicos e } \\
\text { questões para investigações subseqüentes. Em geral, o questionário é adaptado para } \\
\text { cada projeto de desenvolvimento de software em particular, e para o estágio do } \\
\text { projeto. A aplicação de um questionário pode demandar a realização de duas fases: } \\
\text { (1) Fase de Perguntas \& Respostas; (2) Esclarecimento das dúvidas. (UHER \& } \\
\text { TOAKLEY, 1999) ; (CARR et al., 1993) }\end{array}$ \\
\hline 16 & $\begin{array}{l}\text { Abordagem } \\
\text { baseada em Caso } \\
\text { (“Case Based } \\
\text { Approach”) }\end{array}$ & $\begin{array}{l}\text { Técnica baseada na Teoria da Flexibilidade Cognitiva que é capacidade que uma } \\
\text { pessoa tem de diante de uma situação nova ou problema, reestruturar o } \\
\text { conhecimento para resolver a situação ou problema. Desta forma esta técnica utiliza } \\
\text { o caso como centro da abordagem, onde cada caso pode ser decomposto em mini- } \\
\text { casos, e a partir daí, os casos são desconstruídos. (UHER \& TOAKLEY, 1999); } \\
\text { (CARVALHO, 2000) }\end{array}$ \\
\hline
\end{tabular}

Quadro 01 - Técnicas de Identificação de Risco Fonte: Autores

No presente caso, abordaremos de forma mais detalhada a técnica "Brainstorming", por observarmos que tanto na literatura quanto na prática esta técnica vem sendo amplamente aplicada. Deste modo, descreveremos algumas características levantadas na literatura para a aplicação desta técnica.

A primeira característica segundo Nóbrega et al (1997) corresponde a divisão do "Brainstorming" em duas fases, onde na primeira ocorre a geração livre das idéias e na segunda a seleção das melhores idéias.

A segunda característica de acordo com Hicks (apud CHAPMAN, 1998) define o problema e solução aceitável propondo como primeiro passo para o processo do "Brainstorming" um encontro prévio com o "dono" do problema para definir o problema, determinar sua adequação e discutir o que constitui uma solução aceitável. 
Em relação ao Tamanho do Grupo, OSBORN (apud CHAPMAN, 1998) sugere o número de doze participantes na sessão e que o ideal seria a sessão contar com um líder, um auxiliar do líder, cinco membros regulares que formariam o núcleo do grupo e cinco membros convidados.

Para CHAPMAN (1998) o tamanho do grupo terá impacto direto na qualidade das idéias geradas, sendo que o aumento excessivo do grupo poderá reduzir a coesão entre os participantes.

As Características dos Membros do Grupo corresponde a um outro fator importante sendo que o resultado tenderá a ser mais efetivo se os membros formarem um grupo heterogêneo, com diferentes perspectivas do projeto e diferentes personalidades. Por outro lado, sendo o Brainstorming um processo no qual cada indivíduo coloca abertamente sua visão e discute os riscos identificados, tende a sofrer os efeitos negativos dos excessos de personalidade. Assim sendo, o grau de compatibilidade dos participantes, as características da personalidade de cada um, as diferenças hierárquicas e bloqueios emocionais, que poderão influenciar no resultado final, devem sofrer um monitoramento ou orientação a fim de minimizar os desvios causados por ações de interesses ocultos ou necessidades pessoais de cada participante, ou mesmo a perda de motivação devido à longa duração de tempo das sessões. (ibid., 1998).

Na condução da tarefa, os membros do grupo necessitam assumir uma abordagem diferente das posições que cada um assume em seu grupo de trabalho. Da mesma forma, é necessária a clareza na definição dos objetivos e no grau de importância de cada tarefa, além da preparação das sessões e disciplina para conduzi-las. Além disto, OSBORN (apud CHAPMAN, 1998) considera que a técnica deve ser utilizada como suplementar a geração individual de idéias, conferência convencionais e treinamento, e não como técnica substituta. Desta forma ele coloca que a metodologia ideal para busca de idéias seria: (1) geração individual de idéias; (2) "Brainstorming”; (3) geração de idéias em grupo.

Segundo Chapman (1998), uma outra característica é a questão do ambiente. Para o autor todos os grupos trabalham dentro de um ambiente, seja o de um projeto ou subprojeto, e este ambiente impõe certas condições ou restrições na forma como eles operam. Neste aspecto o autor menciona ainda quatro pontos principais, a saber: normas e expectativas, posição do líder, relações entre os grupos e a localização física.

No caso das normas e expectativas, a suas influências no processo do "Brainstorming” são insignificantes. Entretanto, o líder deve evitar monopolizar a sessão, sendo que caso isso ocorra o facilitador (auxiliar do líder) deverá intervir. As relações entre os grupos devem ser gerenciadas para que todos percebam que o processo presta uma importante contribuição aos objetivos do projeto. Por outro lado, não existe restrição quanto à localização física desde que o grupo possa estar afastado das distrações externas e interrupções ou que o arranjo físico esteja arrumado de tal modo que evite a formação de pequenos grupos, que gerariam discussões paralelas fora do enfoque proposto. (ibid., 1998)

Quanto ao critério de efetividade, CHAPMAN (1998) propõe que o mesmo seja estabelecido antes do início do estudo. Por outro lado, para DOWLING \& ST. LOUIS (2000) o número de idéias geradas durante uma sessão de "Brainstorming" é sempre considerada como um indicador de efetividade. Entretanto, como os membros freqüentemente geram idéias duplicadas durante a sessão, o número de idéias geradas sem repetição é mais útil que o total de idéias geradas.

De outro modo, alguns autores abordam a baixa performance da técnica de "Brainstorming". Para PAULUS (1998) a literatura sobre "brainstorming" em grupos foca no processo de geração de idéias e não na qualidade da decisão. Já para 
RIETZSCHEL et al (2006) muitas organizações consideram o "Brainstorming" em grupo como uma técnica efetiva para a geração de um grande número de idéias criativas. Esta popularidade persiste apesar de repetidas descobertas de que o "Brainstorming" individual (ex. grupos nominais), onde as idéias são conciliadas, gera uma quantidade maior e melhor de idéias do que o "Brainstorming" em grupo.

\section{APLICAÇÃO DA TÉCNICA "BRAINSTORMING" EM EMPREENDIMENTOS NA ÁREA DE EXPLORAÇÃO E PRODUÇÃO (E \& P)}

Os projetos que foram analisados neste trabalho são plataformas do tipo flutuante. A escolha deste tipo de plataforma deve-se ao fato de serem projetos atuais de alta complexidade e tecnologia, voltados para a área de exploração e produção de petróleo em águas profundas. Da mesma forma, obtivemos a autorização de uma empresa de grande porte deste setor para procedermos à pesquisa documental dos materiais produzidos durante os workshops de gestão de risco nestes três projetos de engenharia, construção e montagem destas plataformas.

Ao analisarmos os três projetos observamos que a técnica empregada dentre as demais técnicas levantadas para identificação de risco foi a técnica de "Brainstorming". Deste modo, a seguir apresentamos as características gerais de cada um destes projetos.

Projeto A - Consiste na construção de uma plataforma de petróleo do tipo semisubmersível, com 125 metros de comprimento por 110 metros de largura, para operação em uma profundidade de 1200 metros e capacidade de processamento de 180 mil bdp (barris por dia) de óleo e 6 milhões m3/d4 de gás. Sua construção é realizada por empresas contratadas e fiscalizada pela proprietária da plataforma, em um contrato do tipo EPC ("Engineering, Procurement and Construction”). O projeto de construção é composto pelas fases de detalhamento de engenharia, suprimento, construção e montagem, comissionamento e préoperação.

Projeto B - Consiste na conversão de uma plataforma de petróleo do tipo FPSO ("Floating, Production, Storage and Offloading”), para operação em uma profundidade de aproximadamente 1300 metros e capacidade de processamento de 60 mil bdp de óleo. Sua conversão é realizada por empresa contratada e fiscalizada pela proprietária da plataforma, em um contrato do tipo EPC. O projeto de construção é composto pelas fases de detalhamento de engenharia, suprimento, construção e montagem, comissionamento e pré-operação.

Projeto C - Consiste na construção de uma plataforma de petróleo do tipo semi-submersível, com 125 metros de comprimento por 110 metros de largura, para operação em uma profundidade de 1795 metros e capacidade de processamento de 180 mil bdp de óleo e 9,3 milhões m3/d de gás. Sua construção é realizada por empresas contratadas e fiscalizada pela proprietária da plataforma, em um contrato do tipo EPC. O projeto de construção é composto pelas fases de detalhamento de engenharia, suprimento, construção e montagem, comissionamento e pré-operação.

No Quadro 02, apresentamos de forma resumida a aplicação da técnica "Brainstorming" de identificação de risco descrevendo as 11 características verificadas nos projetos acima citados comparando-os entre si e confrontando-os com o preconizado pela Literatura. Seguese uma breve descrição das características avaliadas:

\footnotetext{
${ }^{4} \mathrm{~m}^{3} / \mathrm{d}$ significa metros cúbico por dia 


\begin{tabular}{|c|c|c|c|c|}
\hline & Projeto A & Projeto B & Projeto C & Literatura \\
\hline $\begin{array}{l}\text { Fases do } \\
\text { "Brainstorming" }\end{array}$ & $\begin{array}{l}\text { Duas fases: 1) } \\
\text { apresentação das } \\
\text { idéias; 2) filtro das } \\
\text { melhores idéias. }\end{array}$ & ND & $\begin{array}{l}\text { Duas fases: 1) } \\
\text { apresentação das } \\
\text { idéias; 2) filtro das } \\
\text { melhores idéias. }\end{array}$ & $\begin{array}{l}\text { Duas fases: 1) } \\
\text { apresentação das idéias; 2) } \\
\text { filtro das melhores idéias. } \\
\text { (NÓBREGA apud } \\
\text { MORANO, 2003) }\end{array}$ \\
\hline $\begin{array}{l}\text { Definição do } \\
\text { Problema e Solução } \\
\text { Aceitável }\end{array}$ & $\begin{array}{l}\text { Sem encontro prévio; } \\
\text { Sem sessão inicial de } \\
\text { redefinição do } \\
\text { problema; } \\
\text { Divulgação do } \\
\text { conceito de risco e } \\
\text { gestão de risco. }\end{array}$ & $\begin{array}{l}\text { Sem encontro } \\
\text { prévio; Sem } \\
\text { sessão inicial de } \\
\text { redefinição do } \\
\text { problema. } \\
\text { Divulgação do } \\
\text { conceito de risco e } \\
\text { gestão de risco. }\end{array}$ & $\begin{array}{l}\text { Sem encontro } \\
\text { prévio; Sem sessão } \\
\text { inicial de } \\
\text { redefinição do } \\
\text { problema; } \\
\text { Divulgação do } \\
\text { conceito de risco e } \\
\text { gestão de risco. }\end{array}$ & $\begin{array}{l}\text { Encontro prévio para } \\
\text { definição do problema; } \\
\text { Sessão inicial para } \\
\text { redefinição do problema; } \\
\text { (HICKS apud } \\
\text { CHAPMAN, 1998) } \\
\text { Não cita. }\end{array}$ \\
\hline Tamanho do Grupo & $\begin{array}{l}23 \text { pessoas; } \\
\text { Um único grupo; } \\
\text { Um facilitador para } \\
\text { todo o grupo. }\end{array}$ & $\begin{array}{l}30 \text { pessoas; } \\
\text { Subgrupos de até } \\
8 \text { pessoas; } \\
\text { Um facilitador por } \\
\text { subgrupo. }\end{array}$ & $\begin{array}{l}25 \text { pessoas; } \\
\text { Subgrupos de até } 8 \\
\text { pessoas; } \\
\text { Um facilitador por } \\
\text { subgrupo. }\end{array}$ & $\begin{array}{l}12 \text { pessoas; } \\
\text { Líder, auxiliar, } 5 \text { membros } \\
\text { regulares, } 5 \text { membros } \\
\text { convidados. } \\
\text { (OSBORN apud } \\
\text { CHAPMAN, 1998) }\end{array}$ \\
\hline $\begin{array}{l}\text { Características dos } \\
\text { Membros do Grupo }\end{array}$ & $\begin{array}{l}\text { Diferentes } \\
\text { perspectivas; } \\
\text { Diferentes } \\
\text { personalidades; } \\
\text { Diferentes níveis } \\
\text { hierárquicos; } \\
\text { Posições diferentes } \\
\text { na relação cliente- } \\
\text { fornecedor. }\end{array}$ & $\begin{array}{l}\text { Diferentes } \\
\text { perspectivas; } \\
\text { Diferentes níveis } \\
\text { hierárquicos; } \\
\text { Posições } \\
\text { diferentes na } \\
\text { relação cliente- } \\
\text { fornecedor. }\end{array}$ & $\begin{array}{l}\text { Diferentes } \\
\text { perspectivas; } \\
\text { Diferentes níveis } \\
\text { hierárquicos; } \\
\text { Posições diferentes } \\
\text { na relação cliente- } \\
\text { fornecedor. }\end{array}$ & $\begin{array}{l}\text { Grupo heterogêneo: } \\
\text { diferentes perspectivas e } \\
\text { personalidades } \\
\text { (CHAPMAN, 1998) }\end{array}$ \\
\hline Tempo de Duração & $\begin{array}{l}\text { Evento total: } \\
\text { aproximadamente } 8 \\
\text { horas } \\
\text { Identificação: } \\
\text { aproximadamente } 3 \\
\text { horas }\end{array}$ & $\begin{array}{l}\text { Evento total: } \\
\text { aproximadamente } \\
8 \text { horas } \\
\text { Identificação: } \\
\text { aproximadamente } \\
3 \text { horas }\end{array}$ & $\begin{array}{l}\text { Evento total: } \\
\text { aproximadamente } 8 \\
\text { horas } \\
\text { Identificação: } \\
\text { aproximadamente } 2 \\
\text { horas e } 45 \text { minutos }\end{array}$ & ND \\
\hline Ambiente & $\begin{array}{l}\text { Evitou-se domínio da } \\
\text { sessão por um líder; } \\
\text { localização física } \\
\text { externa ao local de } \\
\text { trabalho; mesma sala; } \\
\text { arrumação em forma } \\
\text { de “U”. }\end{array}$ & $\begin{array}{l}\text { Localização física } \\
\text { externa ao local de } \\
\text { trabalho; demais } \\
\text { pontos não } \\
\text { verificados. }\end{array}$ & $\begin{array}{l}\text { Localização física } \\
\text { externa ao local de } \\
\text { trabalho; mesma } \\
\text { sala; grupo total não } \\
\text { monopolizado. }\end{array}$ & $\begin{array}{l}\text { Normas e expectativas da } \\
\text { organização não } \\
\text { influenciam; o líder não } \\
\text { pode dominar a sessão; o } \\
\text { grupo deve perceber que o } \\
\text { processo contribui para os } \\
\text { objetivos; ambiente livre } \\
\text { de interrupções; } \\
\text { arrumação deve evitar } \\
\text { formação de pequenos } \\
\text { grupos }\end{array}$ \\
\hline Infra-estrutura & $\begin{array}{l}\text { Infra-estrutura } \\
\text { tecnológica. }\end{array}$ & $\begin{array}{l}\text { Infra-estrutura } \\
\text { tecnológica. }\end{array}$ & $\begin{array}{l}\text { Infra-estrutura } \\
\text { tecnológica. }\end{array}$ & $\mathrm{ND}$ \\
\hline Material de Apoio & Distribuído em papel. & $\begin{array}{l}\text { Distribuído em } \\
\text { papel. }\end{array}$ & $\begin{array}{l}\text { Distribuído em } \\
\text { papel. }\end{array}$ & ND \\
\hline Registro das Idéias & $\begin{array}{l}\text { Em planilha } \\
\text { eletrônica. }\end{array}$ & $\begin{array}{l}\text { Em planilha } \\
\text { eletrônica. }\end{array}$ & $\begin{array}{l}\text { Em planilha } \\
\text { eletrônica. }\end{array}$ & ND \\
\hline $\begin{array}{l}\text { Seleção das Melhores } \\
\text { Idéias }\end{array}$ & $\begin{array}{l}\text { Em conjunto com a } \\
\text { qualificação de } \\
\text { riscos. }\end{array}$ & ND & $\begin{array}{l}\text { Em conjunto com a } \\
\text { qualificação de } \\
\text { riscos. }\end{array}$ & $\begin{array}{l}\text { Posterior e independente } \\
\text { da apresentação das idéias } \\
\text { (NÓBREGA apud } \\
\text { MORANO, 2003) } \\
\text { (HICKS apud } \\
\text { CHAPMAN, 1998) }\end{array}$ \\
\hline Efetividade & $\begin{array}{l}\text { Sem critério prévio; } \\
108 \text { riscos } \\
\text { identificados; } 66 \\
\text { riscos selecionados. }\end{array}$ & $\begin{array}{l}\text { Critério prévio não } \\
\text { identificado; } \\
173 \text { riscos } \\
\text { identificados. }\end{array}$ & $\begin{array}{l}\text { Sem critério prévio; } \\
141 \text { riscos } \\
\text { identificados. }\end{array}$ & $\begin{array}{l}\text { Critério prévio } \\
\text { (CHAPMAN, 1998); } \\
\text { Número de idéias geradas } \\
\text { (DOWLING \& ST. } \\
\text { LOUIS, 2000) }\end{array}$ \\
\hline
\end{tabular}

Quadro 2 - Resumo de Comparação dos Projetos com a Literatura

Fonte: Martins, 2006, p. 78 e 79 
Na análise da aplicação da técnica de "Brainstorming" entre os Projetos A, B e $C$ verificamos as seguintes semelhanças.

- A ausência de um encontro prévio para a definição do problema e definição da solução aceitável;

- A composição heterogênea do grupo;

- O tempo de duração total do evento de aproximadamente 8 horas e de aproximadamente 3 horas para a fase de identificação dos riscos;

- A aplicação da técnica em um ambiente externo ao local de trabalho dos participantes;

- A utilização de uma infra-estrutura tecnológica (computador, data-show, etc) para apoiar a condução do evento;

- A distribuição aos participantes de um material de apoio em papel;

- O registro das melhores idéias em planilhas eletrônicas;

- A ausência da definição de um critério de efetividade para medir o sucesso ou fracasso da aplicação do processo.

Da mesma forma, foram observadas entre os Projetos A, B e C as seguintes diferenças:

- Aplicação da técnica em duas fases: Apesar dos Projetos A e C terem aplicado a técnica em duas fases, esta característica não foi possível de ser identificada no Projeto $\mathrm{B}$, devido à ausência desta informação nos documentos pesquisados.

- Tamanho do grupo: os Projetos B e C subdividiram o grupo em grupos menores de até 8 pessoas, mas o Projeto A aplicou a técnica em um grupo único de 23 pessoas;

- Seleção das melhores idéias: os Projetos A e C realizaram a seleção das melhores idéias em conjunto com a qualificação dos riscos, entretanto no Projeto B não foi possível identificar de que forma foi realizada a seleção das melhores idéias, pois não foi encontrada esta informação na documentação pesquisada.

Por outro lado, comparando a aplicação da Técnica "Brainstorming” nos ENGEVISTA, v. 8, n. 2, p. 120-133, dezembro 2006 três projetos com o que preconiza a literatura, verificamos as seguintes similaridades:

- A formação de um grupo heterogêneo de pessoas, com diferentes perspectivas e personalidades.

- A característica do ambiente utilizado para aplicação desta técnica nos três projetos possui similaridade parcial com a literatura, como por exemplo, ela ser aplicada em um ambiente externo ao do trabalho. Entretanto, outros pontos descritos na literatura não puderam ser identificados nos documentos pesquisados.

Dentre os demais pontos semelhantes entre os três Projetos, dois não foram aplicados conforme citado na literatura:

- A definição do problema e da solução aceitável. Hicks (apud CHAPMAN, 1998) propõe como primeiro passo para o processo do "Brainstorming" um encontro prévio com o "dono" do problema para definir o problema, determinar sua adequação e discutir o que constitui uma solução aceitável. Nos projetos pesquisados, nenhum deles considerou esta característica citada na literatura, não definindo assim previamente o problema e a solução aceitável;

- A literatura sugere a adoção prévia de um critério de efetividade que possa medir o sucesso ou fracasso da aplicação do processo. Entretanto, o critério de efetividade da atividade não foi estabelecido ou identificado em nenhum dos três projetos pesquisados.

Dos itens de tempo de duração, infra-estrutura utilizada, material de apoio e forma de registro de idéias, verificados nos três projetos, não encontramos nenhuma sugestão similar na literatura pesquisada.

Da mesma forma, comparando os pontos divergentes entre os três Projetos pesquisados e comparando-os com a literatura observamos que: 
- A aplicação da técnica de "Brainstorming” em duas fases, onde na primeira são apresentadas as idéias (fase criativa) e na segunda são selecionadas as melhores idéias (fase crítica) (NÓBREGA apud MORANO, 2003) ocorreu nos Projetos A e C, entretanto no Projeto $B$ não foi possível identificar esta característica devido a ausência desta informação na documentação pesquisada;

- Quanto ao tamanho do grupo, apesar dos três projetos terem número total de participantes, de 23 a 30 pessoas, superior ao citado pela literatura, de 12 pessoas (OSBORN apud CHAPMAN, 1998), os Projetos B e C subdividiram este total em grupos menores, de até 8 pessoas, um número inferior ao citado pela literatura;

- De acordo com a literatura, na aplicação do "Brainstorming", em um primeiro momento ocorre a listagem de todas as idéias geradas pelo grupo (fase criativa). Num segundo momento faz-se a seleção das melhores idéias geradas nesta listagem (fase crítica) (NÓBREGA apud MORANO, 2003)(HICKS apud CHAPMAN, 1998). Os Projetos A e $C$ apresentaram as mesmas fases descritas na literatura, entretanto a divergência encontrada foi verificada na fase crítica, que diferentemente dos autores foi realizada junto com a fase de qualificação dos riscos.

\section{CONCLUSÃO}

O objetivo deste trabalho foi o de apresentar o processo de identificação de risco e as técnicas mais utilizadas em projetos. Da mesma forma, mostrar como foi aplicada a técnica de identificação de risco "Brainstorming" em três empreendimentos de plataformas de exploração e produção (E \& P) e promover a comparação desta aplicação com a literatura a fim de se verificar as convergências e divergências existentes.

A metodologia utilizada para o levantamento dos dados foi pesquisa documental. A escolha deste tipo de pesquisa foi porque além dos empreendimentos apresentarem alta complexidade e tecnologia, a empresa responsável por estes três projetos de engenharia, construção e montagem destas plataformas é considerada como "top de linha” na área de exploração e produção de petróleo em águas profundas. Da mesma, esta empresa disponibilizou e autorizou a análise documental dos materiais produzidos durante os workshops de gestão de risco para que pudéssemos desenvolver o presente trabalho.

Através da pesquisa de levantamento documental verificou-se que a técnica mais utilizada foi o "Brainstorming". Este resultado se alinha ao encontrado na literatura, que também indica a técnica "Brainstorming" como uma das principais técnicas de identificação de risco aplicadas em projetos. Por outro lado, cabe ressaltar o que vários autores vêm mencionando em relação a pouca utilização das outras técnicas de identificação de risco, por falta de conhecimento.

De acordo com a literatura e a própria análise documental destes três empreendimentos de grande complexidade e importância, constatamos que técnica "Brainstorming" vem sendo amplamente utilizada no Brasil em vários segmentos, o que pode justificar a razão da escolha desta técnica para aplicação nos três projetos estudados. Entretanto, observou-se que as orientações da literatura não foram totalmente seguidas na prática, apesar de algumas etapas terem sido seguidas, o que pode comprometer os resultados obtidos. Além disto, algumas das características apresentadas não foram abordadas na literatura pesquisada, todavia acreditamos que dentre estas algumas são puramente dependentes da tecnologia disponível e da facilidade de utilização desta tecnologia 
por parte dos participantes, não podendo ser identificados como fatores de vantagem. Tome-se como exemplo a utilização de computadores, data-show e planilhas eletrônicas que não garantem um melhor resultado do que um processo conduzido utilizando-se de um "flip-chart". Da mesma forma, a pesquisa traz uma indicação de que a prática de gerenciamento de risco ainda é incipiente no Brasil, e que existe um desconhecimento da aplicação das técnicas de identificação risco conforme especificado pela literatura bem como as de avaliação de risco.

\section{REFERÊNCIAS BIBLIOGRÁFICAS}

AIKEN, M.; KROSP J.; SHIRANI, A.; MARTIN J. Electronic Brainstorming in small and large group. Information \& Management. v. 27, p. 141-149. 1994.

AKINTOYE, Akintola S. MACLEOD, Malcolm J. Risk analysis and Management in construction. International Journal of Project Management, s.l., v. 15, n. 1, p. 31-38, 1997.

BACCARINI, David. Risk Management Australian Style - Theory vs. Practice. In: Project Management Institute Annual Seminars \& Symposium; 2001 Nov 1-10; Nashville, Tennessee, USA.

BERUVIDES, Mario G. Group Decision Support Systems and Consensus Building: Issues in Electronic Media. Computers \& Industrial Engineering, s.l., v. 29, n. 1-4, p. 601-605, 1995.

BOEHM, Barry W. Software risk management: principles and practices. IEEE Software, jan, p. 32-41, 1991.

CARR, Marvin J. et al. Taxonomy-Based Risk Identification. CMU/SEI-93-TR-06, Software Engineering Institute, Carnegie Mellon University, Pittsburgh, Pennsylvania, 1993.

CARVAlHO, Ana Amélia A. A Representação do Conhecimento Segundo a Teoria da Flexibilidade Cognitiva. Portugal: Revista Portuguesa de Educação, v. 13, n. 1, p. 169-184, 2000.

CHAPMAN, Chris B. Project risk analysis and management - PRAM the generic process. International Journal of Project Management, v. 15, n. 5, p. 273-281, 1997.

CHAPMAN, Chris. WARD, Stephen. Why risk efficiency is a key aspect of best practice projects. International Journal of Project Management, n. 22, p. 619-632, 2004.

CHAPMAN, Robert J. The role of system dynamics in understanding the impact of changes to key personnel on design production within construction projects. International Journal of Project Management, v. 16, n. 4, p. 235-247, 1998.

The Effectiveness of Working Group Risk Identification and Assessment Techniques. International Journal of Project Management, v. 16, n. 6, p. 333343, 1998.

The controlling influences on effective risk identification and assessment for construction design management. International Journal of Project Management, v. 19, n. 3, p. 147-160, 2001.

CROWLEY, Mark. Evaluating Influence Diagrams. University of British Columbia, 2004. Disponível em: $<$ http://www.cs.ubc.ca/ crowley/academia/ papers/aiproj.pdf $>$. Acesso em: 22 ago. 2006.

DEY, Prasanta K. OGUNLANA, Stephen

O. Selection and application of risk management tools and techniques for build-operate-transfer projects. Industrial Management \& Data Systems, s.l., v. 104, n. 4, p. 334-346, 2004.

DEY, Prasanta K. Decision support system for risk management: a case study. Management Decision, s.l., v. 39, n. 8, p. 634-649, 2001. 
DOWLING, Karen L. ST. LOUIS, Robert D. Asynchronous implementation of the nominal group technique: is it effective?. Decision Support Systems, s.l., v. 29, p. 229-248, 2000.

HALL, David J. The role of creativity within Best practice manufacturing. Technovation. Great Britain: Elsevier Science Ltd., v.13, n.3, p. 115-121, 1996.

JOHNSON, Pontus et al. Extended Influence Diagrams for Enterprise Architecture Analysis. In: Proceedings of the 10th IEEE International Annual Enterprise Distributed Object Computing Conference. Out. 2006. Disponível em: $<$ http://www.ee.kth.se/php/index.php?actio $\mathrm{n}=$ publications $>$. Acesso em: 22 ago. 2006.

KERZNER, Harold. Project Management: a systems approach to planning, scheduling, and controlling. United States, John Wiley \& Sons, 2001. 1180 p.

MARTINS, Claudia Garrido. Aplicação das Técnicas de Identificação de Risco em Projetos de E \& P. 2006, 93f. Monografia (Pós-Graduação - MBA em Engenharia Econômica e Financeira) - Universidade Federal Fluminense - UFF, Niterói, 2006.

MORANO, Cássia Andréa Ruotolo. Aplicação das Técnicas de Análise de Risco em Projetos de Construção. 2003. 206 f. Dissertação (Mestrado em Engenharia Civil) - Universidade Federal Fluminense - UFF, Niterói, 2003.

NÓBREGA, M. de M.; NETO D. L.; SANTOS, S. R. dos. Uso da técnica de brainstorming para tomada de decisões na equipe de enfermagem de saúde pública. Revista Brasileira de Enfermagem., Brasília, v. 50, n.2, p. 247-256, 1997.

PAULUS, Paul B. Developing consensus about groupthink after all these years. Organizational Behavior and Human Decision Processes, s.l., v. 73, n.2-3, p. 362-374, 1998.

PROJECT MANAGEMENT INSTITUTE (PMI). A Guide to the Project Management
Body of Knowledge: PMBOK guide. 3. ed. Pennsylvania, USA, 2004, 388 p.

RAMOS, Esmeralda. Técnicas de Conducción de Grupos. Universidad Central de Venezuela, s.d..

RAMP - Risk Analysis and Management for Projects. Disponível em:

$<$ http://www.ramprisk.com/>. Acesso em: 04 mar. 2006.

RAZ, T. MICHAEL, E. Use and benefits of tools for project risk management.

International Journal of Project

Management, s.l., v. 19, p. 9-17, 2001.

RIETZSCHEL, Eric F. NIJSTAD, Bernard A. STROEBE, Wolfgang. Productivity is not enough: A comparison of interactive and nominal brainstorming groups on idea generation and selection. Journal of Experimental Social Psychology, s.l., v. 42, n. 2, p. 244-251, 2006.

ROONEY, James J. HEUVEL, Lee N. Vanden. Root Cause Analysis For Beginners. Quality Progress, s.l., p. 45-53, jul. 2004. Disponível em: < http://www.asq.org/pub/qualityprogress/pa st/0704/qp0704rooney.pdf>. Acesso em: 15 ago. 2006.

SABA, Farhad. Synectics... a Brainstorming Tool. San Diego State University. Disponível em: $<$ http://edweb.sdsu.edu/courses/ET650_onl ine/MAPPS/Synectics.html>. Acesso em: 19 nov. 2006.

UHER, Thomas E. TOAKLEY, A. Ray. Risk management in the conceptual phase of a project. International Journal of Project Management, v. 17, n. 3, p. 161169, 1999.

WIKIPEDIA. SWOT Analysis. Disponível em:

http://en.wikipedia.org/wiki/SWOT_Analy sis>. Acesso em: 17 ago. 2006.

WILLIAMS, Terry. A classified bibliography of recent research relating to project risk management. European Journal 
of Operational Research, v. 85, p. 18-38, 1995.

WRIGHT, James Terence Coulter; GIOVINAZZO, Renata Alves. DELPHI Uma ferramenta de apoio ao planejamento prospectivo. Caderno de Pesquisas em Administração. São Paulo, v.1, n.12, 2000. 